\title{
Effects of Artificial Leg Length Discrepancies on the Dynamic Joint Angles of the Hip, Knee, and Ankle During Gait
}

\author{
Yong-Wook Kim, PT, Ph.D • Seung-Yeon Jo • Yeoung-In Byeon • Ji-Ho Kwon • Seok-Hee Im • \\ Su-Hyeon Cheon $\cdot$ Eun-Joo Kim, OT, Ph. $D^{1 \dagger}$
}

Department of Physical Therapy, College of Medical Sciences, Jeonju University ${ }^{1}$ Department of Occupational Therapy, College of Medical Sciences, Jeonju University

Received: December 4, 2018 / Revised: December 11, 2018 / Accepted: January 3, 2019

(c) 2019 J Korean Soc Phys Med

\section{| Abstract |}

PURPOSE: This study examined the dynamic range of motion (ROM) of the hip, knee, and ankle joint when wearing different shoe sole lifts, as well as the limb asymmetry of the range according to the leg length discrepancy (LLD) during normal speed walking.

METHODS: The participants were 40 healthy adults. A motion analysis system was used to collect kinematic ROM data. The participants had 40 markers attached to their lower extremities and were asked to walk on a $6 \mathrm{~m}$ walkway, under three different shoe lift conditions (without an insole, $1 \mathrm{~cm}$ insole, and $2 \mathrm{~cm}$ insole). Visual3D professional software was used to coordinate kinematic ROM data.

RESULTS: Most of the ROM variables of the short limbs were similar under each insole lift condition ( $p>05$ ). In contrast, when wearing a shoe with a $2 \mathrm{~cm}$ insole lift, the long

$\uparrow$ Corresponding Author : Eun-Joo Kim

https://orcid.org/0000-0002-3109-4818

This is an Open Access article distributed under the terms of the Creative Commons Attribution Non-Commercial License (http://creativecommons.org/licenses/by-nc/3.0) which permits unrestricted non-commercial use, distribution, and reproduction in any medium, provided the original work is properly cited. limbs showed significant increases in flexion and extension of the knee joint as well as; plantarflexion, dorsiflexion, pronation, eversion, and inversion of the ankle joint $(\mathrm{p}<.05)$. Of the shoes with the insole lifts, significant differences in all ROM variables were observed between the left and right knees, except for the knee internal rotation $(\mathrm{p}<.05)$.

CONCLUSION: As the insole lift was increased, more ROM differences were observed between the left and right limbs, and the asymmetry of the bilateral lower limbs increased. Therefore, appropriate interventions for LLD are needed because an artificial mild LLD of less than $2.0 \mathrm{~cm}$ could lead to a range of musculoskeletal problems of the lower extremities, such as knee and ankle osteoarthritis.

Key Words: Gait, Joint range of motion, Leg length discrepancy

\section{Introduction}

A leg length discrepancy (LLD), which increases posture and alignment changes, can be affected by congenital or acquired factors[1-3]. Up to $70 \%$ of the population has LLDs, and a discrepancy of more than $2.0 \mathrm{~cm}$ has been observed in at least one out of every 1000 people[4]. LLDs 
are relatively common and can cause many musculoskeletal problems, such as hip osteoarthritis, knee pain, back pain, and scoliosis associated with biomechanical disorders[1]. Previous studies reported that a LLD, as high as $2.5 \mathrm{~cm}$, did not adversely affect the gait or running activities[5,6]. On the other hand, LLDs have a significant association with plantar fasciitis[7], chronic low back pain[8], stress fractures of the lower limbs among runners[9], knee osteoarthritis[10], and functional scoliosis in children[11].

Clear verification of the biomechanical influences of LLD on the neuromuscular system is very important for preventing musculoskeletal problems of the lower limb and improving the walking ability[12,13]. Most LLD studies using shoe sole lifts have used outcome measurements as the qualitative measures of gait[14], pelvic and spine movement[15,16], feet pressure contact pattern and loading of the short leg[17], and peak joint moments[18]. Few studies, however, have examined the relationship between LLD and dynamic joint ranges of the lower extremities using different shoe sole lifts and a three-dimensional (3D) motion analysis system while walking in healthy individuals. Therefore, this study examined the dynamic joint angles of the lower extremities when wearing different shoe sole lifts as well as the limb asymmetry of the range, according to an artificial LLD during free walking.

\section{Methods}

\section{Participants}

This study recruited 40 healthy volunteers who met the selection criteria and consented to participate. The study population consisted of eight males and 32 females. The participants provided written informed consent after being given a detailed explanation of the experimental process. The study was conducted in accordance with the principles of the Declaration of Helsinki and was approved by the institutional review board of Jeonju University (jjIRB170615-HR-2017-0609).
Participants who could walk freely $10 \mathrm{~m}$ with an even gait were recruited for this study. Participants who had $\geq 1.0 \mathrm{~cm}$ of LLD, and any musculoskeletal problems of the lower limbs were excluded. The leg length was measured using a tape measure between the medial malleolus and the anterior superior iliac spine in the supine position[19]. The mean ages, heights, and weights of all participants were $20.7 \pm 1.4$ years, $164.2 \pm 7.3 \mathrm{~cm}$, and $61.4 \pm 10.5 \mathrm{~kg}$, respectively.

\section{Instrumentation and procedure}

Three dimensional (3D) dynamic range of motion (ROM) data of the hip, knee, and ankle joints were obtained using a Vicon Motion Capture System (Vicon Inc., Oxford, England) with six T10 cameras operating at a $100 \mathrm{~Hz}$ sampling rate. Nexus 1.8.5 software (Vicon, Oxford, England) was used to process the captured ROM data in three planes. To calibrate six T10 cameras, a $7.5 \mathrm{~cm}$ calibration wand was used.

Forty reflective markers $(1.4 \mathrm{~cm})$ were attached bilaterally to the participants' anterior superior iliac spine, posterior superior iliac spine, greater trochanters, malleoli, and feet. The participants had cluster markers attached bilaterally to the shank and thigh segments according to the six-degrees-of-freedom (6DOF) model[20] (Fig. 1). Static calibration data were captured from each participant to produce a hybrid model for later analysis of the dynamic ROM of the lower extremities during the walking trials. Following this static calibration capture, the calibrated anatomical system technique was used to measure the changes in the kinematic ROM data of the lower limbs were measured while the participants walked freely wearing a normalized shoe, a shoe with a $1 \mathrm{~cm}$ soft insole lift (Cubio, JKGOLD, Korea), and a shoe with a $2 \mathrm{~cm}$ soft insole lift (Cubio, JKGOLD, Korea) (Fig. 2). To obtain the kinematic ROM data of the lower extremity joints and segments, the participants walked along a $6 \mathrm{~m}$ walkway at their preferred walking speed. The participants performed 


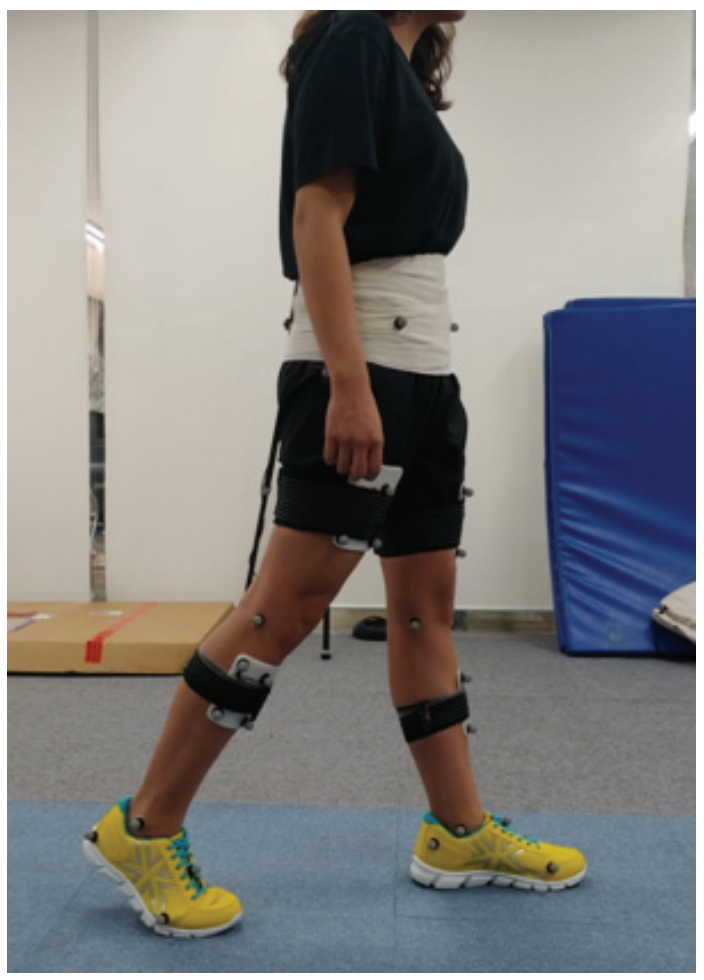

Fig. 1. Reflective and cluster markers setting of the lower extremities for dynamic motion capture

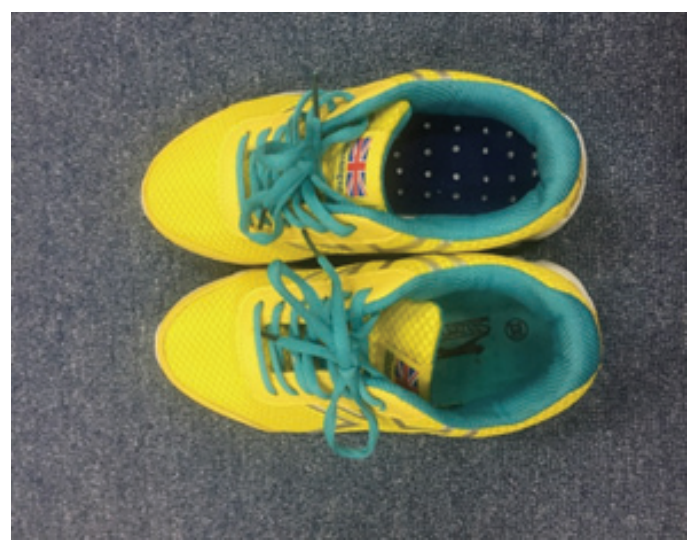

Fig. 2. Normalized shoe with a $2 \mathrm{~cm}$ insole lift-inserted on the right side

a total of 8 to 10 walk trials. The order in which the shoes were worn (normalized shoe, $1 \mathrm{~cm}$ insole lift-inserted right shoe, or $2 \mathrm{~cm}$ insole lift-inserted right shoe) was assigned randomly before the walking trials began.

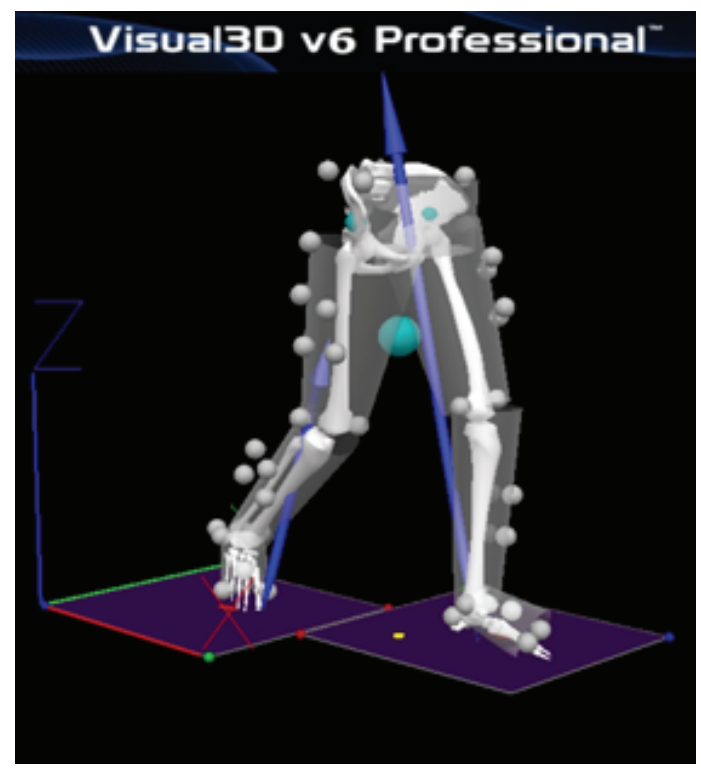

Fig. 3. Calibrated Anatomical System Technique to measure range of motion of the ankle and foot joints, using a Visual3D program

After data collection using the Vicon system and Nexus program, Visual3D analysis software (C-Motion, Rockville, MD, USA) was used to obtain the final lower limbs ROM results and graph reports of each joint of the lower extremities. The kinematic data were low-pass filtered with a fourth-order Butterworth filter and a cutoff frequency of $6 \mathrm{~Hz}$. Visual3D produced a visual representation of the bone segments in space that enabled the related joint angles to be calculated (Fig. 3).

\section{Data analysis}

All analyses were conducted using SPSS (ver. 21.0; IBM Corp., Armonk, NY, USA). To confirm the normal distribution of the data, the Kolmogorov-Smirnov test was used. The maximum peak ROM values of each joint of the lower limbs were used for comparison analysis according to each shoe condition. In addition, the maximum ROMs of the ankle joint were used for the analysis based on the angle at $25 \%$ (mid-stance) of the total gait cycle of each shoe condition. One-way repeated measures 
Table 1. Mean and standard deviation of the left hip, knee, and ankle joints in each shoe condition using one-way repeated measures ANOVA with a Bonferroni's correction

\begin{tabular}{|c|c|c|c|c|c|}
\hline Left & Maximal ROM (degree) & Standard shoe & $\begin{array}{l}\text { Shoe with } \\
1 \mathrm{~cm} \text { insole lift }\end{array}$ & $\begin{array}{c}\text { Shoe with } \\
2 \mathrm{~cm} \text { insole lift }\end{array}$ & $\mathrm{p}$ \\
\hline \multirow{6}{*}{ Hip } & Flexion & $35.871 \pm 6.668$ & $34.471 \pm 6.870$ & $35.301 \pm 9.034$ & .412 \\
\hline & Extension & $7.475 \pm 6.244$ & $7.293 \pm 5.767$ & $9.289 \pm 10.967$ & .317 \\
\hline & Adduction & $3.430 \pm 3.102$ & $3.455 \pm 3.404$ & $2.441 \pm 2.960$ & $.002 *$ \\
\hline & Abduction & $9.207 \pm 3.551$ & $8.888 \pm 3.531$ & $9.954 \pm 3.178$ & $.008 *$ \\
\hline & Internal Rotation & $7.371 \pm 8.572$ & $6.717 \pm 8.046$ & $8.060 \pm 11.765$ & .319 \\
\hline & External Rotation & $6.548 \pm 8.566$ & $6.142 \pm 9.280$ & $7.941 \pm 12.692$ & .607 \\
\hline \multirow{6}{*}{ Knee } & Flexion & $53.463 \pm 6.021$ & $52.753 \pm 6.888$ & $53.281 \pm 6.604$ & .592 \\
\hline & Extension & $5.851 \pm 5.085$ & $1.894 \pm 5.934$ & $2.001 \pm 5.604$ & .963 \\
\hline & Adduction & $7.234 \pm 3.216$ & $4.377 \pm 3.180$ & $4.390 \pm 3.289$ & .867 \\
\hline & Abduction & $.211 \pm 3.724$ & $2.234 \pm 3.721$ & $1.941 \pm 3.288$ & .629 \\
\hline & Internal Rotation & $2.028 \pm 7.265$ & $1.335 \pm 5.677$ & $1.854 \pm 5.432$ & .617 \\
\hline & External Rotation & $15.591 \pm 7.377$ & $11.230 \pm 5.452$ & $10.391 \pm 5.562$ & .314 \\
\hline \multirow{6}{*}{ Ankle } & Plantarflexion & $33.372 \pm 4.247$ & $32.445 \pm 4.852$ & $30.140 \pm 6.644$ & .062 \\
\hline & Dorsiflexion & $10.255 \pm 4.220$ & $11.466 \pm 4.721$ & $11.719 \pm 8.051$ & .623 \\
\hline & Supination & $4.943 \pm 5.324$ & $4.804 \pm 5.121$ & $4.356 \pm 4.563$ & .590 \\
\hline & Pronation & $6.911 \pm 5.367$ & $6.854 \pm 5.428$ & $7.064 \pm 5.101$ & .937 \\
\hline & Eversion & $10.984 \pm 4.350$ & $11.091 \pm 4.928$ & $11.101 \pm 6.891$ & .946 \\
\hline & Inversion & $3.411 \pm 3.547$ & $3.511 \pm 3.678$ & $3.090 \pm 5.937$ & .677 \\
\hline
\end{tabular}

${ }^{*} p<.05$

ANOVA with a Bonferroni's correction were used to determine the effects of each shoe condition. Paired t-tests were performed to compare the left limb (short leg) and right limb (long leg) differences of each joint angle. Differences were considered significant at the $a=.05$ level.

\section{Results}

Most ROM variables of the left short limb with each insole lift condition during walking were similar except for hip adduction and abduction ROM ( $\mathrm{p}>$.05) (Table 1). In contrast, the hip adduction ROM of the right long limb when wearing a shoe insole lifts showed significant differences compared to that without a shoe insole lift
( $>$.05) (Table 2). In addition, the ankle ROM values were significantly different when wearing shoe insole lifts compared to that without a shoe insole lift ( $\mathrm{p}<05$ ) (Table 2). The right knee ROM showed significant increases in the ROM of flexion, extension, and internal rotation with 2 $\mathrm{cm}$ insole lifts, compared to the other shoe conditions $(\mathrm{p}<.05) \quad($ Table 2). Significant increases in ankle plantarflexion, dorsiflexion, pronation, eversion, and inversion ROM were also observed with $2 \mathrm{~cm}$ insole lifts compared to the normalized shoe conditions $(\mathrm{p}<.05)$ (Table 2). The ankle supination ROM showed significant decreases with the $2 \mathrm{~cm}$ insole compared to the other shoe conditions $(\mathrm{p}<.05)$ (Table 2).

A comparison of the ROM variables between the short 
Table 2. Mean and standard deviation of the right hip, knee, and ankle joints in each shoe condition using one-way repeated measures ANOVA with a Bonferroni's correction

\begin{tabular}{|c|c|c|c|c|c|}
\hline Right & Maximal ROM (degree) & Standard shoe & $\begin{array}{c}\text { Shoe with } \\
1 \mathrm{~cm} \text { insole lift }\end{array}$ & $\begin{array}{c}\text { Shoe with } \\
2 \mathrm{~cm} \text { insole lift }\end{array}$ & $\mathrm{p}$ \\
\hline \multirow{6}{*}{ Hip } & Flexion & $36.156 \pm 6.741$ & $38.791 \pm 10.143$ & $40.029 \pm 8.500$ & .054 \\
\hline & Extension & $7.089 \pm 7.820$ & $4.227 \pm 11.844$ & $6.336 \pm 8.537$ & .304 \\
\hline & Adduction & $2.664 \pm 2.901$ & $2.566 \pm 2.961$ & $3.910 \pm 3.201$ & $.001^{*}$ \\
\hline & Abduction & $9.565 \pm 2.820$ & $10.080 \pm 2.643$ & $8.810 \pm 2.855$ & $.001^{*}$ \\
\hline & Internal Rotation & $8.927 \pm 8.021$ & $9.911 \pm 8.444$ & $11.777 \pm 11.939$ & .470 \\
\hline & External Rotation & $6.559 \pm 8.192$ & $5.507 \pm 7.800$ & $7.340 \pm 12.177$ & .234 \\
\hline \multirow{6}{*}{ Knee } & Flexion & $59.154 \pm 7.702$ & $71.567 \pm 8.134$ & $73.404 \pm 6.088$ & $.003^{*}$ \\
\hline & Extension & $6.690 \pm 6.733$ & $7.311 \pm 7.030$ & $8.041 \pm 6.519$ & $.032 *$ \\
\hline & Adduction & $7.526 \pm 3.167$ & $8.136 \pm 3.897$ & $7.544 \pm 3.248$ & .134 \\
\hline & Abduction & $.393 \pm 3.162$ & $.850 \pm 3.281$ & $.136 \pm 3.280$ & .059 \\
\hline & Internal Rotation & $.519 \pm 4.893$ & $.082 \pm 4.877$ & $1.402 \pm 5.307$ & $.043 *$ \\
\hline & External Rotation & $17.088 \pm 5.802$ & $18.376 \pm 6.236$ & $17.470 \pm 6.254$ & .124 \\
\hline \multirow{6}{*}{ Ankle } & Plantarflexion & $32.446 \pm 3.774$ & $33.029 \pm 4.645$ & $35.041 \pm 3.377$ & $.001 *$ \\
\hline & Dorsiflexion & $9.590 \pm 4.063$ & $10.766 \pm 3.557$ & $12.746 \pm 3.932$ & $.002 *$ \\
\hline & Supination & $4.233 \pm 5.281$ & $2.035 \pm 4.502$ & $.217 \pm 4.455$ & $.000 *$ \\
\hline & Pronation & $7.288 \pm 5.402$ & $8.877 \pm 5.019$ & $9.772 \pm 5.130$ & $.001 *$ \\
\hline & Eversion & $10.007 \pm 3.871$ & $10.788 \pm 3.846$ & $11.888 \pm 4.445$ & $.002 *$ \\
\hline & Inversion & $2.00 \pm 3.43$ & $3.654 \pm 3.080$ & $5.893 \pm 3.810$ & $.001 *$ \\
\hline
\end{tabular}

${ }^{*} \mathrm{p}<.05$

and long lower limbs using a paired t-test showed no significant differences between the left and right ROM variables when wearing normalized shoes only $(\mathrm{p}>.05)$ (Table 3, 4, 5). The right hip flexion ROM showed significant increases with $1 \mathrm{~cm}$ insole lifts, compared to the left side $(\mathrm{p}<.05)$ (Table 3). Significant differences in the hip ROM in flexion, extension, adduction, and internal rotation were observed with the $2 \mathrm{~cm}$ insole lifts ( $<<.05$ ) (Table 3). All ROM variables of the knee joint showed significant differences compared to that without a shoe insole lift $(\mathrm{p}<.05)$ (Table 4). Significant differences in plantarflexion, supination, pronation, and inversion were observed between the left and right ankle ROM when the participants wore shoes with $2 \mathrm{~cm}$ insole lifts $(\mathrm{p}<.05)$ (Table 5).

\section{Discussion}

This study examined the changes in the ROM and symmetry of the lower limbs during walking when artificial LLDs of $0 \mathrm{~cm}, 1 \mathrm{~cm}$, and $2 \mathrm{~cm}$ shoe insole lifts were worn. The results showed that the long limb (the right lower extremity) condition produced significant differences in many ROM variables compared to the short limb conditions, when wearing different shoe lifts. The long limbs showed greater hip flexion, knee flexion and extension, as well as ankle plantarflexion, dorsiflexion, pronation, eversion, and inversion angles. On the other hand, the hip abduction and ankle supination angles were decreased significantly in the long limbs. A comparison 
Table 3. Mean and standard deviation of the left and right hip joint

\begin{tabular}{|c|c|c|c|c|}
\hline Condition & Maximal ROM (degree) & Left hip & Right hip & $\mathrm{p}$ \\
\hline \multirow{6}{*}{$\begin{array}{c}\text { Normal } \\
\text { shoe }\end{array}$} & Flexion & $35.866 \pm 6.668$ & $36.160 \pm 6.743$ & .702 \\
\hline & Extension & $7.477 \pm 6.243$ & $7.088 \pm 7.824$ & .627 \\
\hline & Adduction & $3.427 \pm 3.100$ & $2.656 \pm 2.900$ & .229 \\
\hline & Abduction & $9.209 \pm 3.554$ & $9.574 \pm 2.821$ & .654 \\
\hline & Internal Rotation & $7.365 \pm 8.564$ & $8.925 \pm 8.017$ & .366 \\
\hline & External Rotation & $6.550 \pm 8.574$ & $6.555 \pm 8.188$ & .990 \\
\hline \multirow{6}{*}{$\begin{array}{c}\text { Shoe with } \\
1 \mathrm{~cm} \text { insole } \\
\text { lift }\end{array}$} & Flexion & $34.474 \pm 6.872$ & $38.791 \pm 10.141$ & $.013^{*}$ \\
\hline & Extension & $7.288 \pm 5.770$ & $4.231 \pm 11.854$ & .094 \\
\hline & Adduction & $3.455 \pm 3.400$ & $2.565 \pm 2.955$ & .164 \\
\hline & Abduction & $8.889 \pm 3.534$ & $10.081 \pm 2.643$ & .077 \\
\hline & Internal Rotation & $6.721 \pm 8.052$ & $9.910 \pm 8.435$ & .054 \\
\hline & External Rotation & $6.137 \pm 9.280$ & $5.511 \pm 7.801$ & .643 \\
\hline \multirow{6}{*}{$\begin{array}{c}\text { Shoe with } \\
2 \mathrm{~cm} \text { insole } \\
\text { lift }\end{array}$} & Flexion & $35.301 \pm 9.032$ & $40.029 \pm 8.504$ & $.000^{*}$ \\
\hline & Extension & $9.287 \pm 10.970$ & $6.344 \pm 8.543$ & $.000^{*}$ \\
\hline & Adduction & $2.444 \pm 2.964$ & $3.907 \pm 3.203$ & $.022 *$ \\
\hline & Abduction & $9.950 \pm 3.181$ & $8.813 \pm 2.856$ & .110 \\
\hline & Internal Rotation & $8.055 \pm 11.768$ & $11.768 \pm 11.941$ & $.014 *$ \\
\hline & External Rotation & $7.940 \pm 12.686$ & $7.344 \pm 12.178$ & .660 \\
\hline
\end{tabular}

$* \mathrm{p}<.05$

of each insole lift during gait revealed a significant difference in; both hip joint adduction and abduction angles, which is believed to compensate for the pelvic inclination due to the LLD. The knee and ankle joints of the long legs showed significant joint angle changes in response to changes in the height of the insole, but the short leg knee and ankle joint angles did not show any significant differences. These results showed greater angular changes in the long limb joints with higher shoe lifts. Although no previous studies compared these results directly, Resende et al.[21] reported that of 19 healthy individuals, the participants preferred strategies to increase the short limb functional length (increased rearfoot plantarflexion and inversion angles and decreased knee and hip flexion angles) and reduce the long limb length (increased rearfoot dorsiflexion and eversion angles and knee and hip flexion angles). A previous study produced similar results to the present study in most ROM variables except for rearfoot plantarflexion and inversion ROM, possibly because the rearfoot ROMs were not measured in this study. The shorter leg showed smaller hip and knee flexion ranges and the longer leg showed larger hip and knee ranges in this study. This means that when limping caused by the LLD occurred, the compensatory changes in joint angle were observed mainly on the long leg side. These results show that individuals use adaptations to strategically lengthen the short leg and shorten the long leg.

A comparison of each matched ROM variable between the left and right hip joints showed no significant differences between any of the ROM variables of the left and right hip joints, when wearing normalized shoes. On the other hand, as the height of the insole lift was increased, 
Table 4. Mean and standard deviation of the left and right knee joint

\begin{tabular}{|c|c|c|c|c|}
\hline Condition & Maximal ROM (degree) & Left knee & Right knee & $\mathrm{p}$ \\
\hline \multirow{6}{*}{$\begin{array}{c}\text { Normal } \\
\text { shoe }\end{array}$} & Flexion & $53.464 \pm 6.018$ & $59.145 \pm 7.700$ & .671 \\
\hline & Extension & $5.847 \pm 5.090$ & $6.688 \pm 6.730$ & .444 \\
\hline & Adduction & $7.228 \pm 3.221$ & $7.528 \pm 3.170$ & .809 \\
\hline & Abduction & $.210 \pm 3.717$ & $.390 \pm 3.161$ & .631 \\
\hline & Internal Rotation & $2.034 \pm 7.266$ & $.522 \pm 4.892$ & .103 \\
\hline & External Rotation & $15.589 \pm 7.377$ & $17.087 \pm 5.801$ & .378 \\
\hline \multirow{6}{*}{$\begin{array}{c}\text { Shoe with } \\
1 \mathrm{~cm} \text { insole } \\
\text { lift }\end{array}$} & Flexion & $52.750 \pm 6.892$ & $71.573 \pm 8.132$ & $.000^{*}$ \\
\hline & Extension & $1.888 \pm 5.934$ & $7.314 \pm 7.028$ & $.000 *$ \\
\hline & Adduction & $4.378 \pm 3.18$ & $8.143 \pm 3.902$ & $.000^{*}$ \\
\hline & Abduction & $2.234 \pm 3.720$ & $.852 \pm 3.277$ & $.000^{*}$ \\
\hline & Internal Rotation & $1.342 \pm 5.683$ & $.083 \pm 4.879$ & .091 \\
\hline & External Rotation & $11.234 \pm 5.447$ & $18.381 \pm 6.243$ & $.000 *$ \\
\hline \multirow{6}{*}{$\begin{array}{c}\text { Shoe with } \\
2 \mathrm{~cm} \text { insole } \\
\text { lift }\end{array}$} & Flexion & $53.280 \pm 6.597$ & $73.400 \pm 6.087$ & $.000^{*}$ \\
\hline & Extension & $2.000 \pm 5.595$ & $8.042 \pm 6.516$ & $.000^{*}$ \\
\hline & Adduction & $4.394 \pm 3.290$ & $7.543 \pm 3.250$ & $.000 *$ \\
\hline & Abduction & $1.936 \pm 3.289$ & $.142 \pm 3.281$ & $.000 *$ \\
\hline & Internal Rotation & $1.845 \pm 5.441$ & $1.401 \pm 5.312$ & .621 \\
\hline & External Rotation & $10.392 \pm 5.563$ & $17.466 \pm 6.254$ & $.000 *$ \\
\hline
\end{tabular}

$* \mathrm{p}<.05$

significant ROM differences were observed between both sides in many ROM motions, and the bilateral symmetry became worse. The symmetry results for the right and left knee and ankle ROM variables were also similar to those for the hip joints. In particular, shoes with 1 or $2 \mathrm{~cm}$ insole lifts resulted in significant changes in all knee ROM variables, except for internal rotation ROM. These results suggest that of the joints of the lower limbs, the knee joint is most sensitive to a height difference of the LLD during gait. The prominent aspect of the change in bilateral ankle joints using an insole lift was observed in the ROM of ankle supination and pronation. Blake and Ferguson[22] reported more rearfoot eversion on the long limb, which is in accordance with the study results showing increased ankle pronation on the long limb when wearing insole lifts. In particular, a significant decrease in ankle supination and a significant increase in ankle pronation occurred in the long leg, and biomechanical adaptation was made to achieve equilibrium with a short opposite leg when wearing a $2 \mathrm{~cm}$ insole lift. Although most people have mild LLD less than $2.0 \mathrm{~cm}$, there is no consensus regarding the effects of mild LLD on gait[23,24]. This study examined the effects of artificial mild LLD on the kinematics of the lower limbs during gait. Some of the results agree with a previous study, which reported the biomechanical effects on gait in subjects with true LLD[22].

This study had some limitations. This study was conducted on healthy individuals, who were mostly younger than the general age. Therefore, it is difficult to generalize these results to those with actual LLD. Although artificial application of LLD clearly demonstrated kinematic changes in the ROM of hip, knee, and ankle joints during gait, 
Table 5. Mean and standard deviation of the left and right ankle joint

\begin{tabular}{|c|c|c|c|c|}
\hline Condition & Maximal ROM (degree) & Left ankle & Right ankle & $\mathrm{p}$ \\
\hline \multirow{6}{*}{$\begin{array}{l}\text { Normal } \\
\text { shoe }\end{array}$} & Plantarflexion & $33.374 \pm 4.244$ & $32.454 \pm 3.766$ & .114 \\
\hline & Dorsiflexion & $10.263 \pm 4.218$ & $9.591 \pm 4.064$ & .070 \\
\hline & Supination & $4.941 \pm 5.322$ & $4.232 \pm 5.280$ & .402 \\
\hline & Pronation & $6.910 \pm 5.373$ & $7.288 \pm 5.404$ & .617 \\
\hline & Eversion & $10.977 \pm 4.3527$ & $10.012 \pm 3.870$ & .064 \\
\hline & Inversion & $3.410 \pm 3.551$ & $2.001 \pm 3.434$ & .053 \\
\hline \multirow{6}{*}{$\begin{array}{c}\text { Shoe with } \\
1 \mathrm{~cm} \text { insole } \\
\text { lift }\end{array}$} & Plantarflexion & $32.454 \pm 4.854$ & $33.033 \pm 4.654$ & .122 \\
\hline & Dorsiflexion & $11.464 \pm 4.724$ & $10.766 \pm 3.563$ & .060 \\
\hline & Supination & $4.801 \pm 5.123$ & $2.042 \pm 4.501$ & $.000^{*}$ \\
\hline & Pronation & $6.844 \pm 5.434$ & $8.877 \pm 5.022$ & $.022 *$ \\
\hline & Eversion & $11.089 \pm 4.930$ & $10.785 \pm 3.845$ & .690 \\
\hline & Inversion & $3.512 \pm 3.677$ & $3.652 \pm 3.081$ & .837 \\
\hline \multirow{6}{*}{$\begin{array}{c}\text { Shoe with } \\
2 \mathrm{~cm} \text { insole } \\
\text { lift }\end{array}$} & Plantarflexion & $30.141 \pm 6.636$ & $35.044 \pm 3.383$ & $.000 *$ \\
\hline & Dorsiflexion & $11.720 \pm 8.047$ & $12.75 \pm 3.928$ & .461 \\
\hline & Supination & $4.361 \pm 4.563$ & $.221 \pm 4.456$ & $.000^{*}$ \\
\hline & Pronation & $7.055 \pm 5.104$ & $9.770 \pm 5.132$ & $.000^{*}$ \\
\hline & Eversion & $11.101 \pm 6.888$ & $11.888 \pm 4.454$ & .381 \\
\hline & Inversion & $3.091 \pm 5.940$ & $5.892 \pm 3.809$ & $.011^{*}$ \\
\hline
\end{tabular}

$* \mathrm{p}<.05$

the results could provide important information for future LLD research on the kinematics and kinetics effect using shoe insole lifts. Further studies will be needed to examine the effects of these biomechanical variables in patients with LLD.

\section{Conclusion}

The results showed significant differences in the ROMs of the long limb during walking with each shoe condition, compared to the short limb without insole lifts. As the insole lift height was increased, more ROM differences in many ROM variables were observed between the left and right limbs, and asymmetry of bilateral lower limbs was increased. Therefore, appropriate interventions for LLD will be needed because an artificial mild LLD of less than $2.0 \mathrm{~cm}$ can cause a range of musculoskeletal problems of the lower extremities, such as knee and ankle osteoarthritis.

\section{Acknowledgements}

This study was supported by the National Research Foundation of Korea (NRF) grant funded by the Ministry of Science and ICT (No. 2018R1C1B5042645).

\section{References}

Azizan NA, Basaruddin KS, Salleh AF. The Effects of Leg Length Discrepancy on Stability and KinematicsKinetics Deviations: A Systematic Review. Appl Bionics Biomech. 2018:5156348.

Beattie P, Isaacson K, Riddle DL, et al. Validity of derived 
measurements of leg-length differences obtained by use of a tape measure. Phys Ther. 1990;70(3):150-7.

Bennell K, Matheson G, Meeuwisse W, et al. Risk factors for stress fractures. Sports Med. 1999;28(2):91-122.

Blake RL, Ferguson HJ. Correlation between limb length discrepancy and asymmetrical rearfoot position. $\mathrm{J}$ Am Podiatr Med Assoc. 1993;83(11):625-33.

Collins TD, Ghoussayni SN, Ewins DJ, et al. A six degrees-of-freedom marker set for gait analysis: repeatability and comparison with a modified Helen Hayes set. Gait Posture. 2009;30(2):173-80.

Defrin R, Ben Benyamin S, Aldubi RD, et al. Conservative correction of leg-length discrepancies of $10 \mathrm{~mm}$ or less for the relief of chronic low back pain. Arch Phys Med Rehabil. 2005;86(11):2075-80.

Goel A, Loudon J, Nazare A, et al. Joint moments in minor limb length discrepancy: a pilot study. Am J Orthop (Belle Mead NJ). 1997;26(12):852-6.

Gross RH. Leg length discrepancy in marathon runners. Am J Sports Med. 1983;11(3):121-4.

Guichet JM, Spivak JM, Trouilloud P, et al. Lower limb-length discrepancy. An epidemiologic study. Clin Orthop Relat Res. 1991;272:235-41.

Gurney B, Mermier C, Robergs R, et al. Effects of limb-length discrepancy on gait economy and lower-extremity muscle activity in older adults. J Bone Joint Surg Am. 2001;83-A(6):907-15.

Hellsing AL. Leg length inequality. A prospective study of young men during their military service. Ups J Med Sci. 1988;93(3):245-53.

Kakushima M, Miyamoto K, Shimizu K. The effect of leg length discrepancy on spinal motion during gait: three-dimensional analysis in healthy volunteers. Spine (Phila Pa 1976). 2003;28(21):2472-6.

Kaufman KR, Miller LS, Sutherland DH. Gait asymmetry in patients with limb-length inequality. J Pediatr Orthop. 1996;16(2):144-50.

Khamis S, Carmeli E. Relationship and significance of gait deviations associated with limb length discrepancy: A systematic review. Gait Posture. 2017;57:115-23.

Mahmood S, Huffman LK, Harris JG. Limb-length discrepancy as a cause of plantar fasciitis. J Am Podiatr Med Assoc. 2010;100(6):452-5.

Needham R, Chockalingam N, Dunning D, et al. The effect of leg length discrepancy on pelvis and spine kinematics during gait. Stud Health Technol Inform. 2012;176:104-7.

O'Toole GC, Makwana NK, Lunn J, et al. The effect of leg length discrepancy on foot loading patterns and contact times. Foot Ankle Int. 2003;24(3):256-9.

Raczkowski JW, Daniszewska B, Zolynski K. Functional scoliosis caused by leg length discrepancy. Arch Med Sci. 2010;6(3):393-8.

Resende RA, Kirkwood RN, Deluzio KJ, et al. Biomechanical strategies implemented to compensate for mild leg length discrepancy during gait. Gait Posture. 2016;46:147-53.

Resende RA, Kirkwood RN, Deluzio KJ, et al. Mild leg length discrepancy affects lower limbs, pelvis and trunk biomechanics of individuals with knee osteoarthritis during gait. Clin Biomech (Bristol, Avon). 2016;38: 1-7.

Sabharwal S, Kumar A. Methods for assessing leg length discrepancy. Clin Orthop Relat Res. 2008;466(12): 2910-22.

Walsh M, Connolly P, Jenkinson A, et al. Leg length discrepancy--an experimental study of compensatory changes in three dimensions using gait analysis. Gait Posture. 2000;12(2):156-61.

Wretenberg P, Hugo A, Broström E. Hip joint load in relation to leg length discrepancy. Med Devices (Auckl). 2008;1:13-8.

Zeitoune G, Nadal J, Batista LA, et al. Prediction of mild anatomical leg length discrepancy based on gait kinematics and linear regression model. Gait Posture. 2019;67:117-21. 
